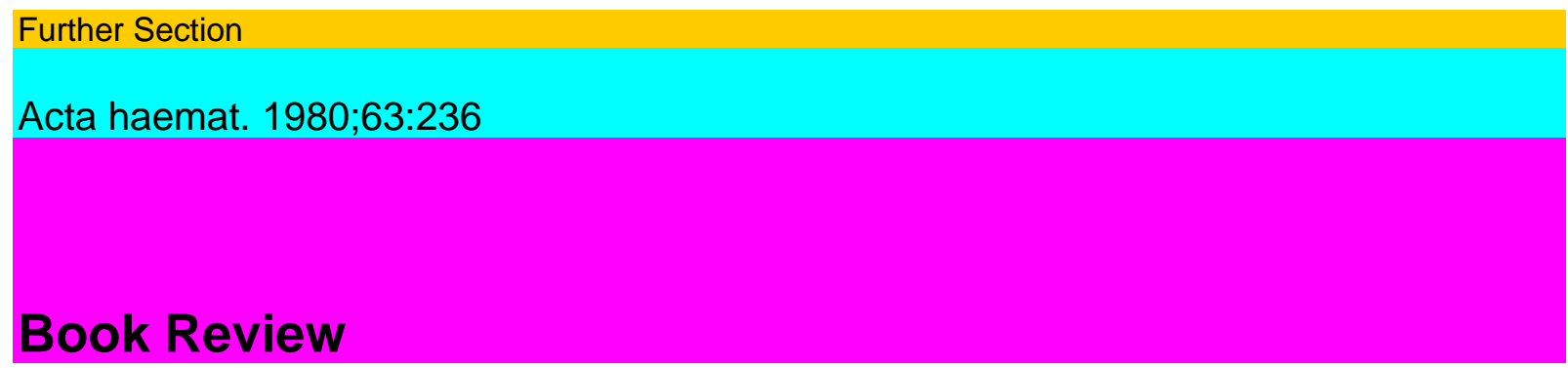

\title{
F. Heckner
}

Praktikum der mikroskopischen Hämatologie;

4. Aufl. Urban \& Schwarzenberg, München 1978 XI + 121 pp., 105 fig. in 204 ein- und mehrfarbigen Teilbildern; DM 36.-ISBN 3-541-01004-5

Nebst den vielen hämatologischen Büchern nimmt auch die Anzahl der morphologischen Wer-ke in letzter Zeit wieder zu. Man muss sich deshalb fragen, wo der Platz dieses «Praktikums der mikroskopischen Hämatologie» von Fritz Heckner ist. Die Erkennung der Blutkrankheiten findet, wie der Autor im Vorwort selber schreibt, in der ärztlichen Sprechstunde, am Krankenbett und im Laborato-rium statt. Die fortgeschrittene Mechanisierung der Medizin hat jedoch bisher die richtige Deutung und exakte Unterscheidung von Blut- und Knochen-markszellen durch das Auge des Untersuchers nicht verdrängt. Das vorliegende Werk ist eine Sammlung von Bildern verschiedenster normaler und pathologischer Zellen von Blutbild und Kno-chenmark. Es ist deshalb als «morphologische Stütze» gut geeignet für das Routinelabor, vor al-lem kleinerer Spitäler und grösserer Praxen, wo seltene Krankheitsbilder nicht jeden Tag diagno-stiziert werden. Die Einteilung des Buches ist übersichtlich, der Untersucher wird die gesuchten Zellen rasch finden. Die Erklärung der Bilder ist einfach, klar und stichhaltig. Am Schlusse des Buches findet sich eine kurze Übersicht der wichtig-sten Färbetechniken. Obwohl der Autor erklärt, dass er sich den neuesten Gegebenheiten angepasst hat, ist die Nomenklatur teilweise etwas veraltet. So werden beispielsweise klassische Echinozyten als Akanthozyten bezeichnet (p. 42), und recht häu-fig findet man den veralteten Ausdruck «Para-myelo- oder Paralymphoblasten». Ferner fehlt die neue Internationale Einteilung der Leukämien. Es ist ausserdem schade, dass zum Teil Schwarzweiss-bilder verwendet wurden, wo Farbabbildungen mehr aussagen würden. Etwas unglücklich und meines Erachtens auch überflüssig ist die Anlei-tung zur Befunderhebung von Knochenmarkpunk-taten. Der Autor versucht hier aufgrund symbo-lisch dargestellter Zellen einen Überblick über die Knochenmarksbefunde der einzelnen Erkrankun-gen zu geben. Bis man sich jedoch diese Symbole gemerkt hat, ist man durch Konsultation der Farbabbildungen einfacher und schneller zum Ziel ge-kommen. Diese sind nämlich meist sehr scharf, deutlich und gut in der Farbe.

Trotz der geringen Einschränkungen ist dieses «Praktikum der mikroskopischen Hämatologie», welches nur Hinweise auf die morphologische Diagnostik, nicht aber auf die zugrundeliegenden Krankheitsbilder geben will, für den praktisch täti-gen Arzt und das Labor sehr geeignet.

G. F. Riedler, Luzern 